\title{
Competitive interactions in Drosophila melanogaster IV. Chromosome assay
}

\author{
P. D. S. Caligari* and \\ Kenneth Mather
}

\author{
Department of Genetics, University of Birmingham \\ P.O. Box 363, Birmingham B15 2TT, U.K.
}

\begin{abstract}
The technique of chromosome assay, using chromosome substitution lines, has been refined in Drosophila melanogaster to provide a powerful method of investigating the distribution, amongst the chromosomes, of the underlying genetical determinants, particularly in relation to chaeta characters. In the present study this technique is extended to investigate differences in competitive ability. The genotypes used were two inbred lines chosen as showing differences in competitive ability based on a survey of such lines derived from a large laboratory population. The character followed was $p_{a}$, the proportion (transformed into angles) of eggs which successfully developed into adults. It was found that chromosome III carried gene differences affecting both aggression and response but showing no good evidence of interaction with one another or with genes on other chromosomes. The gene differences on chromosome I, on the other hand, showed no main effect on either aggression or response but a clear interaction with chromosome II in affecting response. Chromosome II also showed a main effect on aggression, though only when associated with one of the two genetically marked lines with which the substitution lines were compared. The implications of these findings are discussed.
\end{abstract}

\section{INTRODUCTION}

The Texas laboratory population of Drosophila melangaster was started from 30 inseminated females caught in the wild in late 1965 and has been maintained in this Department as a cage population since early 1966 . It has been shown to carry substantial amounts of genetic variability not only for external features, notably several chaeta characters (Barnes and Kearsey, 1970; Kearsey and Barnes, 1970; Mather and Hanks 1978; Caligari and Mather, 1980), but also allozyme polymorphism (Minawa and Birley, 1978), ADH enzyme activity and protein level (Birley, Couch and Marson, 1981) and DNA polymorphism (Birley, 1984). A number of inbred lines extracted from the population proved to be of value in analysing the properties of the genes responsible for this variation, and since such lines produce eggs of uniform, or near uniform, genotype they also offered the possibility of testing for genetic variation in respect of competitive ability; and of measuring and analysing any such variation that

\footnotetext{
* Present address: Department of Agricultural Botany, Plant Science Laboratories, University of Reading, Whiteknights, P.O. Box 221, Reading RG6 2AS, U.K.
}

might be found. In 1981, therefore, two experiments of a preliminary nature where undertaken to test the lines for genetic differences in competitive ability. Sixteen of them were used in the first experiment and were tested in monoculture and duoculture with the $F_{1}$ from $y^{2} 27$ and $y^{2} 28$, which were the inbred lines 27 and 28 into which the gene $y^{2}$ (Mather and Caligari, 1983) had been introduced as a marker. Nine of these lines were assessed again in a similar series of tests, in which however the duocultures were with $y^{2} 28$. The results of these experiments were not published at the time partly because the methodology for their full analysis had not then been devised, but they left no doubt that genetic variation for competitive ability existed in the Texas population.

The analysis of this variation has been approached in two ways. First, three of the lines (numbers 1, 5 and 15) were chosen as material for an investigation of competitive behaviour in triocultures, which involved raising and analysing all the three possible duocultures as well as the triocultures themselves (Caligari and Mather, 1984). Behaviour in these duocultures was analysed by Mather and Caligari (1983) using a newly developed means of separating and measuring the 
effects of individuals in exerting selective pressure on their fellows (the aggression or $a$ component of the competitive relationship) and the response of the individual to the pressure so exerted on it (the response or $r$ component). It was shown that $a$ and $r$ could vary simultaneously, but there was no indication that they did so in a correlated fashion. This line of investigation was taken further by Eggleston (1985) using eight of the inbred lines all marked with $\mathrm{y}^{2}$ and comparing them with samples taken from the Texas population itself. He showed that the lines displayed substantial variation for both $a$ and $r$, and again there was no evidence of correlation in the distribution of the two components. As Eggleston emphasised, the two aspects of competition must be separately adjustable by natural selection. It is noteworthy too that the population samples showed a greater value of $a$ than all of the eight inbred lines and a lower value of $r$ than all but one of them.

The second approach to the analysis of the variation in competitive ability was through the use of sets of substitution lines to assay the contributions made by each of the three major chromosomes to the differences between a pair of truebreeding lines. In the past such assays have been used chiefly for the analysis of differences in chaeta characters, but in principle they can be applied to any measurable character, including competitive ability, where however its use requires a further extension of the analytical methodology for obtaining separate estimates of the effects of aggression and response. Such a chromosome assay experiment, begun in 1979 , is the subject of the present report.

\section{THE EXPERIMENT}

The two inbred lines chosen as the basis for this experiment were lines 7 and 28 , but for the sake of brevity the latter will be denoted hereinafter as line 8 . Both are, of course, wild-type but the preliminary experiments had shown that they differed widely in their competitive abilities in respect of the character $p_{a}$, the proportion (transformed into angles) of eggs which have successfully developed into adults. For intra-genotypic competition in monocultures, line 8 showed the highest value of all the 16 tested, whereas line 7 gave no indication of any such competition: its regression of $p_{a}$ on density of eggs in fact showed no significant departure from zero in either of the two preliminary tests. The set of eight substitution lines comprising all the possible true breeding combinations of the three major chromosomes from lines 7 and 8 were constructed using the methods and stocks described by Mather and Caligari (1983). The lines produced will be referred to as $777,778,787,788$, $877,878,887,888$, the order of the numerals indicating the origins of their chromosomes, I, II and III. For comparison the parental lines 7 and 8 were included along with the eight substitution lines in the experimental assessment of competitive ability.

The cultures were all raised in vials with a basis of agar gel to each of which $45 \mathrm{mg}$ of live yeast had been added. The reference density of eggs per culture was 120 , and $x$ took values of $0,-30,-60$, and -90 , so giving densities of $120,90,60$ and 30 eggs of the sole genotype in monocultures. These densities were chosen on the basis of earlier experiments. Caligari (1980) showed that, as might be expected, $p_{a}$ does not show a linear response to increasing density over all possible values. The range of densities used here was therefore chosen such that the response would be expected to approximate to linearity and has been employed in other reported experiments (e.g., Caligari and Mather, 1984). It should be noted, however, that this assumption is not critical to the methods of analysis used, which include a test for the presence of such effects. The duocultures that were also raised used marked versions of lines 7 and 8 , (referred to as $\mathrm{y}^{2} 7$ and $\mathrm{y}^{2} 8$ ) as associates. Monocultures of $y^{2} 7$ and $y^{2} 8$ were also raised. Thus 12 series of monocultures were raised, (the eight substitution lines together with lines 7 and 8 , and lines $y^{2} 7$ and $y^{2} 8$ ) and 20 series of duocultures (the eight substitution lines and lines 7 and 8 , each with $\mathrm{y}^{2} 7$ and $y^{2} 8$ ) as shown in table 1 . The whole experiment was carried out in duplicate and the values of $p_{a}$ used in the analysis were each the mean of the pair of corresponding values, one from each block. The error variance of these means $\left(V_{E}\right)$ is thus half the mean square difference between twin values, in the calculation of which a correction was made for the overall difference between the duplicate blocks.

The results were divided into 12 sets for the purpose of calculating the regression coefficients measuring competition values. Following the principles described by Mather and Caligari (1981), each set was based on a single genotype used as the indicator, whose response to competitive pressures was being measured. Thus the sets based on each of the eight substitution lines and also the two parental lines included three series of observations (summarised in table 1), one from the monocultures of that line, a second from its 
Table 1 Experimental details in terms of the lines used, the number of eggs to initiate each type of culture and the regressions carried out. The whole experiment was raised in duplicate

\begin{tabular}{|c|c|c|c|}
\hline \multirow[b]{2}{*}{ Genotypes } & \multicolumn{3}{|l|}{ Number of eggs } \\
\hline & Monocultures & $\begin{array}{l}\text { Duocultures } \\
\text { with } y^{2} 7 \text { at } \\
90,60,30\end{array}$ & $\begin{array}{l}\text { Duocultures } \\
\text { with } y^{2} 8 \text { at } \\
90,60,30\end{array}$ \\
\hline \multicolumn{4}{|l|}{ Wild-type lines } \\
\hline $\begin{array}{l}\text { Parental lines } \\
7(\text { Texas } 7) \\
8(\text { Texas } 28)\end{array}$ & $\begin{array}{l}120,90,60,30 \\
120,90,60,30\end{array}$ & $\begin{array}{l}30,60,90 \\
30,60,90\end{array}$ & $\begin{array}{l}30,60,90 \\
30,60,90\end{array}$ \\
\hline $\begin{array}{l}\text { Substitution lines } \\
\begin{array}{lll}7 & 7 & 7 \\
7 & 7 & 8 \\
7 & 8 & 7 \\
7 & 8 & 8 \\
8 & 7 & 7 \\
8 & 7 & 8 \\
8 & 8 & 7 \\
8 & 8 & 8\end{array}\end{array}$ & $\begin{array}{c}\text { somes } X, I I, I I I) \\
120,90,60,30 \\
120,90,60,30 \\
120,90,60,30 \\
120,90,60,30 \\
120,90,60,30 \\
120,90,60,30 \\
120,90,60,30 \\
120,90,60,30\end{array}$ & $\begin{array}{l}30,60,90 \\
30,60,90 \\
30,60,90 \\
30,60,90 \\
30,60,90 \\
30,60,90 \\
30,60,90 \\
30,60,90\end{array}$ & $\begin{array}{l}30,60,90 \\
30,60,90 \\
30,60,90 \\
30,60,90 \\
30,60,90 \\
30,60,90 \\
30,60,90 \\
30,60,90\end{array}$ \\
\hline $\begin{array}{l}\text { Marked lines (wit) } \\
y^{2} 7\left(y^{2} \text { Texas } 7\right) \\
y^{2} 8\left(y^{2} \text { Texas } 28\right)\end{array}$ & $\begin{array}{l}\text { ker) } \\
120,90,60,30 \\
120,90,60,30\end{array}$ & $\begin{array}{l}--- \\
---\end{array}$ & $\begin{array}{l}--- \\
---\end{array}$ \\
\hline
\end{tabular}

Regression analyses

(a) Wild-type as indicator and marked line as associate.

Data on wild-type line from: Monoculture

Duocultures with $\mathrm{y}^{2} 7$ and $\mathrm{y}^{2} 8$

(giving 3 regression lines, $b_{m}$ and $2 b_{d}$ 's)

Number of such regression sets: 10 ( 8 substitution and 2 parental lines)

(b) Marked line as indicator and wild-type line as associate.

Data on marked line from: Monoculture

Duocultures with parental lines 7 and 8

Duocultures with $777,778,787,788$, $877,878,887,888$.

(giving 11 regression lines, $b_{m}$ and $10 b_{d}$ 's)

Number of such regressions sets: 2 ( 2 marked lines $y^{2} 7$ and $\left.y^{2} 8\right)$

It should be noted that the equations of estimation are set in detail by Mather and Caligari, 1981

duocultures with $y^{2} 7$, used as associate, and the third from the duoculture series with $y^{2} 8$. The remaining two sets, also noted in table 1 , were based on $y^{2} 7$ and $y^{2} 8$, respectively, used as indicators and each of these included eleven series of observations; the monoculture series and ten duoculture series in each of which one of the ten lines, eight substutition and two parental, provided the associate. Each of the twelve monoculture series thus provided data for only one set, while each duoculture series provided data for two sets according to which of the two genotypes it comprised was treated as indicator and which as associ- ate (see Mather and Caligari, 1981). Within each set a straight regression line was fitted to each of the series it contained, subject to the constraint that all these lines must pass through the same point at the reference density where $x=0$, and all 120 eggs were of the indicator genotype. Where the indicator was one of the ten wild type genotypes, each set yielded three such regression lines: one from the monoculture series whose slope is $b_{m}$, and one from each of the duoculture series with $\mathrm{y}^{2} 7$ and $\mathrm{y}^{2} 8$, with slopes of $b_{d 7}$ and $b_{d 8}$ respectively. The two sets with $\mathrm{y}^{2} 7$ and $\mathrm{y}^{2} 8$, respectively, as indicators each yielded eleven such regression 
lines, one with a slope denoted by $b_{m}$ from the monoculture series and ten others $\left(b_{d}\right.$ 's) from the ten wild-types respectively used as associates.

Mather and Caligari (1981) set out a general method for estimating the regression coefficients and the value of $p_{a}$, denoted by $e$, on which all the regression lines converge in such a set, using a least squares technique. This method has subsequently been used and extended in a number of studies (e.g., Mather and Caligari, 1983; Powell et al., 1985; Eggleston, 1985; Caligari and Powell, 1986; Hill et al., 1987a, b). As given originally by Mather and Caligari (1981) the general formula for the matrix using their least squares fitting contained some errors of sign; but a corrected version is to be found in Mather et al. (1982). It should be noted that both these papers refer to the parameter now termed $e$ as $a$. This should not be confused with $a$ as redefined by Mather and Caligari (1983) and now used here. The two specific matrices, one for the ten sets where the ten wild-type genotypes were used as indicators, and the other for two sets where the marked lines were so used, are shown in table 2.

The goodness of fit with the data of this model using straight regression lines converging at the reference density, was tested (again following Mather and Caligari, 1981) by calculating the values of $p_{a}$ expected for each point in each series, using the estimated $e$ and the appropriate $b$ values. The differences between the expected and observed values are squared and summed. The $S(O-E)^{2}$ corresponds to a number of degrees of freedom found as the number of observations less the number of parameters fitted, and the mean square which is obtained is tested for significance against

Table 2 The information matrix $\left(\mathbf{J}^{-1}\right)$ for the estimation of $e$, $b_{m}$ and $b_{d}$ 's in sets of data where the indicator is (a) one of the two parental or eight substitution lines, and (b) one of the two tester lines

(a)

$$
\begin{gathered}
{\left[\begin{array}{rrr}
44,100 & -630 & -630 \\
-630 & 17 & 9 \\
-630 & 9 & 17
\end{array}\right]} \\
\text { all } \div 100,800
\end{gathered}
$$

$\left[\begin{array}{crrrr}6300 & -630 & -630 & \ldots & -630 \\ -630 & 29 & 9 & \ldots & 9 \\ -630 & 9 & 29 & \ldots & 9 \\ \vdots & \vdots & \vdots & & \vdots \\ -630 & 9 & 9 & \ldots & 29\end{array}\right]$

all $\div 252,000$
$V_{E}$. This test yielded no indication that the fit of the model was inadequate for the ten sets of data in which the wild type lines were indicators, or for that set in which $y^{2} 7$ was the indicator, but the value of the $\operatorname{MS}(O-E)^{2}$ was significantly greater than $V_{E}$ for the test in which $\mathrm{y}^{2} 8$ was the indicator. $\operatorname{MS}(O-E)^{2}$ has therefore been used to provide a more appropriate $V_{E}$ as the basis for finding the error variance of the various parameters estimated from this set of results.

The regression coefficients, $b_{m}$ and $b_{d}$, measure the rate of change of expression of the character, $p_{a}$, on the density of eggs of the indicator genotype, $120+x$, and as such measure the impact of competition on the indicator. Denoting a given wildtype line by $\mathrm{L}$ and a given marker line by $\mathrm{T}$, we can, following Mather et al. (1982), obtain from a monoculture series $c_{\mathrm{LL}}=-b_{m}$ as a measure of the response of an $\mathrm{L}$ individual to the competitive pressure exerted on it by individuals of like phenotype. At the same time, each of the duoculture series with $\mathrm{T}$ in the same set gives $c_{\mathrm{LT}}=b_{d}-b_{m}$ which provides a measure of the $\mathrm{L}$ individuals response to the pressure exerted by $\mathrm{T}$ individuals. Similarly $c_{\mathrm{TT}}=-b_{m}$ from the monocultures in sets where $\mathrm{T}$ is the indicator genotype, $c_{\mathrm{TL}}=b_{d}-b_{d m}$ from a duoculture series in the same set measures the response of $\mathrm{T}$ individuals to the pressure from L. As shown by Mather and Caligari (1983), taken together the two pairs of $c$ values provide the material for separating $a$, the difference in pressure, or aggression, between the two genotypes, and $r$ their difference in response to pressure, providing that $a$ and $r$ are additive, which can also be tested in the same analysis. Our present aim, however, is to estimate differences in competitive values between two lines 7 and 8 which cannot be compared directly because both are wild-type and cannot be distinguished from one another in duocultures; to analyse these into contributions made to $a$ and $r$ by the three major chromosomes; and to do so by comparison using the two marked testerlines as intermediaries. The analytical methodology for estimating $a$ and $r$, where the two components can readily be distinguished one from another, has been described in detail by Mather and Caligari (1983). The present case, using tester lines, requires an extension of this methodology, as we shall see.

\section{RESULTS AND ANALYSIS}

The $c$ values yielded by the twelve sets of data, distinguished from one another by the twelve different genotypes used as indicators, are set out 
Table 3 The values of $e$ and the various $c$ 's for the two parental and eight substitution lines. The relevant variance ( $V$ ) is given at the foot of each column

\begin{tabular}{|c|c|c|c|c|c|c|}
\hline Lines & $e$ & $c_{\mathrm{LL}}$ & $c_{\mathrm{LT} 1}$ & $c_{\mathrm{LT} 2}$ & $c_{\mathrm{T} 1 \mathrm{~L}}$ & $c_{\mathrm{T} 2 \mathrm{~L}}$ \\
\hline \multicolumn{7}{|c|}{ Parental } \\
\hline 7 & $22 \cdot 455$ & 0.026388 & 0.021679 & $0 \cdot 210071$ & -0.033809 & -0.056953 \\
\hline \multicolumn{7}{|c|}{ Substitution } \\
\hline 777 & $11 \cdot 188$ & 0.014030 & -0.035345 & 0.047024 & $0 \cdot 106774$ & 0.040261 \\
\hline 788 & $11 \cdot 917$ & $0 \cdot 268734$ & 0.047333 & $0 \cdot 142619$ & $0 \cdot 176322$ & 0.056077 \\
\hline 877 & $16 \cdot 198$ & 0.027446 & -0.106452 & 0.087928 & 0.094857 & 0.024714 \\
\hline 878 & 16.906 & $0 \cdot 161271$ & 0.059143 & $0 \cdot 127619$ & 0.088655 & 0.048964 \\
\hline 887 & $11 \cdot 284$ & 0.035309 & -0.137810 & -0.064250 & $0 \cdot 132060$ & -0.060096 \\
\hline 888 & $16 \cdot 209$ & $0 \cdot 144389$ & -0.065690 & $0 \cdot 105405$ & $0 \cdot 170893$ & 0.069547 \\
\hline \multicolumn{7}{|c|}{ Testers } \\
\hline & & $c_{\mathrm{TT}}$ & 0.046308 & 0.080522 & & \\
\hline & & $V$ & 0.0019583 & 0.0020826 & & \\
\hline
\end{tabular}

in table 3. The error variances of these $c$ values are shown, set by set, in the bottom row of the table. From the ten sets with wild-type indicators and that in which $\mathrm{y}^{2} 7$ was so used their error variances are based on $V_{p a}=11.611565$; derived from the differences between duplicate observations; in the case of the set with $\mathrm{y}^{2} 8$ as indicator $V_{p a}=24.961959$ since $(O-E)^{2}$ was used as the basis for the reason given earlier. The error variances of the c's was found from $V_{p a}$ using the appropriate elements of the relevant matrix given in table 2, as described by Mather et al. (1982).

The values of $e$ for the twelve sets are also given in table 3 , but these need not detain us for long, since, although $e$ must reflect the competitive interactions of the individuals in the cultures, they can tell us nothing directly about the competitive values: $e$ is the estimated value of $p_{a}$ at a single density, whereas competition is measured by the rate of change of $p_{a}$ on density. Also the value of $e$ will reflect the effect of agencies which, while affecting fitness, do not depend for their action on population density. At the same time, the $e$ values of substitution lines $L_{777}$ and $L_{888}$ are both intermediate between those of the parental lines $\mathrm{L}_{7}$ and $\mathrm{L}_{8}$, and what is more differ in the opposite direction, so showing that some erosion of the original genetical differences between $\mathrm{L}_{7}$ and $\mathrm{L}_{8}$ must have taken place during the construction of the substitution lines-as indeed is commonly the case (see, for example, Caligari and Mather, 1975). The $c$ values also show evidence of erosion, as we shall see later. It is interesting to note that the relation- ships among the $e$ values also show, when an orthogonal analysis is applied to the substitution lines, that while all the major chromosomes make a significant contribution to their difference, there is no evidence of interaction between them in exerting their effects. In contrast such inter-chromosome interaction is found in a corresponding analysis of the effects on competition, to which we must now turn.

\section{Monocultures}

The values of $c_{\mathrm{LL}}$ in table 3 show clear differences between the substitution lines in the way they react to change in density. Indeed, $\mathrm{L}_{788}$ at one extreme shows nearly 20 times the rate of change of $L_{777}$ at the other extreme, despite their closely similar values of $e$. These differences are analysed further in table 4 , where by use of the familiar seven orthogonal comparisons their departures from the overall mean have been broken down into the main effects of the three chromosomes, the three first order interactions of the chromosomes in displaying their effects, and the second order interaction among them, from which, together with the mean also shown in the table, the eight $c_{\mathrm{LL}}$ 's are built up. Thus the difference in $c_{\mathrm{LL}}$ value attributable to chromosome $\mathrm{I}$ is found as $\frac{1}{8}\left(c_{777}+c_{778}+c_{787}+c_{788}-\right.$ $\left.c_{877}-c_{878}-c_{887}-c_{888}\right)$. Its positive sign indicates that in this experiment the chromosome from $\mathrm{L}_{8}$ made a smaller contribution to the competitive value than did its homologue from $\mathrm{L}_{7}$, though in this particular case the difference is not significant and so is attributable to sampling variation. 
Table 4 Above The means and sets of seven orthogonal comparisons among the eight substitution lines in respect of $c_{\mathrm{LL}}, c_{\mathrm{LT}}$ and $c_{\mathrm{TL}}$. All $\times 10^{2}$.

Below The corresponding means and half-differences of the two parental lines. All $\times 10^{2}$.

The variance $\times 10^{4}\left(V_{c}\right)$ of the orthogonal comparisons and half-differences is shown at the foot of each column. These also apply to the mean for $c_{\mathrm{LL}}$ and $c_{\mathrm{LT}}$, but not for $c_{\mathrm{TL}}$

\begin{tabular}{|c|c|c|c|c|c|}
\hline Comparison & $c_{\mathrm{LL}}$ & $c_{\mathrm{LT} 1}$ & $c_{\mathrm{LT} 2}$ & $c_{\mathrm{TIL}}$ & $c_{\mathrm{T} 2 \mathrm{~L}}$ \\
\hline \multicolumn{6}{|c|}{ Substitution Lines } \\
\hline I & $1 \cdot 0011$ & $2 \cdot 3524$ & $1 \cdot 5138$ & 0.9052 & $1 \cdot 0413$ \\
\hline II & $-4 \cdot 6192$ & 0.6726 & 0.4978 & -1.8734 & 0.9471 \\
\hline III & $-4 \cdot 6722$ & $-3 \cdot 7586$ & $-3 \cdot 3246$ & $-1 \cdot 7632$ & $-2 \cdot 4633$ \\
\hline I $\times$ II & $-4 \cdot 8444$ & $-3 \cdot 2321$ & $-3 \cdot 8620$ & $1 \cdot 1097$ & -0.6586 \\
\hline I $\times$ III & $1 \cdot 4006$ & $2 \cdot 1842$ & 1.9091 & -0.9475 & $1 \cdot 3840$ \\
\hline II $\times$ III & $1 \cdot 1535$ & -0.0860 & 1.6430 & 0.6543 & 1.6455 \\
\hline$I \times I I \times I I I$ & 1.7719 & $2 \cdot 2509$ & -1.6061 & -0.4716 & 0.9894 \\
\hline Mean & $10 \cdot 2117$ & -3.9179 & 7.9314 & 13.0668 & $3 \cdot 1195$ \\
\hline$V_{c}$ & 2.4479 & $2 \cdot 3039$ & $2 \cdot 3039$ & $1 \cdot 1519$ & $2 \cdot 4764$ \\
\hline \multicolumn{6}{|c|}{ Parental Lines } \\
\hline Half-diff. & $-18 \cdot 1711$ & $-8 \cdot 1166$ & $1 \cdot 3214$ & $-8 \cdot 5536$ & $-4 \cdot 8810$ \\
\hline Mean & 20.8099 & $10 \cdot 2846$ & $19 \cdot 6857$ & $5 \cdot 1727$ & -0.8144 \\
\hline$V_{c}$ & $9 \cdot 7915$ & $9 \cdot 2155$ & $9 \cdot 2155$ & 4.6078 & $9 \cdot 9055$ \\
\hline
\end{tabular}

Similarly, the negative sign of the component attributable to chromosome II shows that here $\mathrm{L}_{7}$ was making a smaller contribution than $\mathrm{L}_{8}$. The negative value of $c_{\mathrm{LL}}$ for the $\mathrm{I} \times \mathrm{II}$ interaction shows that the parental combinations of chromosomes I and II (i.e., 77 and 88) contributed less to intragenotypic competition than did the cross combinations, 78 and 87 . It should be noted that in this and subsequent tables, and also in the text, the $c$ values are shown $\times 10^{2}$ and the $V_{c}$ are correspondingly shown $\times 10^{4}$. Since the $c_{\mathrm{LL}}$ values of table 3 are all from different sets of data and hence are independent of each other, the variance of the seven orthogonal components as well as that of the mean, which is also shown in table 4 , is one eighth the value of the eight single values of table 3. Application of this variance to the component values of table 4 , shows that components II, III and I $\times$ II make significant contributions to the differences in competition values between $\mathrm{L}_{7}$ and $\mathrm{L}_{8}$, whereas there is no evidence that either the main effect of chromosome I or any of the remaining three interactions does so.

These findings stem solely from the monoculture series and hence from the competition of individuals with their fellows of like genotype. Following Mather and Caligari (1983) in assuming that the effects of the aggressive pressure they exert (a) and their individual responses to that pressure ( $r$ ) are additive, the $c_{\mathrm{LL}}$ values and their components provide information on $a+r$, and tell us nothing of whether the differences we have found are due to differences in $a$, differences in $r$, or some combination of the two. To separate the differences attributable to $a$ and $r$, we must turn to the data from duocultures. Before doing so, however, there arises from the monocultures a further point to note.

As shown in table $3, \mathrm{~L}_{7}$ gives $c_{\mathrm{LL}}=0.026388$ while that from $L_{8}$ is 0.389809 , giving a mean value of 0.208099 and a half-difference of $18 \cdot 1711$. Now the half-difference displays the effects only of loci at which the two parental lines had different alleles, and indeed will be the sum of half the differences in effects of the two alleles at each relevant locus: it will not even include the average effects of the two alleles at a locus where the two alleles differ. Furthermore the differences may be in opposite directions, and so tend to balance out. The mean on the other hand will include not only the effect of all the relevant loci at which the alleles do not differ, but also the average effects at loci where they do. The fact that $c_{\mathrm{LL}}$ for $\mathrm{L}_{7}$ is so small, with the consequence that the half-difference between the two lines is not much less than the mean, tells us not only that there can be but few genes affecting competitive value that are common to the two lines, but also that $\mathrm{L}_{8}$ carries all, or nearly all, the alleles increasing competition value, and $\mathrm{L}_{7}$ all or nearly all the alleles decreasing it, at the loci where the two parental lines differ.

\section{Estimating the difference in a and $r$}

To separate the differences in the values of $a$ and $r$ we now turn to the duocultures and the values 
of $c_{\mathrm{LT}}$ and $c_{\mathrm{TL}}$ that they yield. Where a tester line, say $T_{1}$, is used as associate with all eight of the substitution lines used as indicators, the same set of orthogonal components as used in the analysis of the monocultures can again be applied to the $c_{\mathrm{LT1}}$ values from the duocultures. Since, however, the associate is the same in all the duocultures in question, $a$ has the same value in all of them and hence will cancel out in the orthogonal comparisons. These will thus yield estimates of differences in the $r$ elements of substitution lines, as set out in table 4. Similarly, in duocultures where the substitution lines are used as associates with a common indicator, say $T_{1}$ again, the orthogonal comparisons will yield estimates of the differences in the $a$ elements in the competition values of the substitution lines, again as set out in table 4. So, on the assumption of additivity of $a$ and $r$ values, the sum of $c_{\mathrm{LT}}$ should equal the value given by the corresponding comparisons in the analysis of $c_{\mathrm{LL}}$ derived from the monocultures, apart from sampling variation. Furthermore, the $a$ and $r$ elements of the differences in competition value among the substitution lines should, apart from sampling variation, be the same whether using $T_{1}$ or $T_{2}$ as a tester.

The expectations can be tested, but valid tests of significance require efficient estimates of the $a$ and $r$ elements. These can be obtained for each of the orthogonal comparisons in turn by bringing the relevant values of $c_{\mathrm{LL}}, c_{\mathrm{TL}}$ and $c_{\mathrm{LT}}$ into the same analysis, and deriving the estimates of $a$ and $r$ by weighted least squares analysis, the weights being $I \stackrel{=}{=} 1 / V$ where $V$ is the estimate of error variation to which the value of $c$ is subject in the set of data in question. The error variances of $c_{\mathrm{LL}}$, $c_{\mathrm{LT} 1}$ and $c_{\mathrm{LT} 2}$ from each substitution line are found using the methods described in earlier papers. In the present case the matrix in table 2(a) is used and $V_{c}$ is $17 V_{E} / 100,800$ for $c_{\mathrm{LL}}$, while for $c_{\mathrm{LT1}}$ and $c_{\mathrm{LT} 2}$ it is $2(17-9) V_{E} / 100,800$. Since $V_{E}=$ 11.611565 for these sets of data we obtain $V_{c}=$ 0.0019583 for $c_{\mathrm{LL}}$ and 0.0018431 for $c_{\mathrm{LT} 1}$ and $c_{\mathrm{LT} 2}$ as shown in table 3 , or 19.583 and 18.431 when $\times 10^{4}$. Since the sets of data for the eight substitution lines used as indicators are independent of each other, these variances are divided by 8 to give the error variances of the orthogonal components as set out in table 3 . The same error variances apply to the mean values given for $c_{\mathrm{LL}}, c_{\mathrm{LT} 1}$ and $c_{\mathrm{LT} 2}$ in table 4 .

The derivation of the error variances for the orthogonal components of $c_{\mathrm{T} 1 \mathrm{~L}}$ and $c_{\mathrm{T} 2 \mathrm{~L}}$ is more complex since in each case $b_{m}$ and the eight $b_{d}$ 's for the substitution lines were all obtained from the same set of data using the matrix in table 2(b), though the data for $T_{1}$ and $T_{2}$ are independent of each other. This makes no complication in respect of the error variances appropriate to the $c_{\mathrm{TT}}$ and $c_{\mathrm{TL}}$ of the substitution lines themselves, but since in each case only a single estimate has been made of $b_{m}$ this single value has been used in calculating $c_{\mathrm{TL}}=b_{d}-b_{m}$ for all eight substitution lines. Thus when the seven orthogonal comparisons are calculated, $b_{m}$ cancels out exactly as before leaving as the estimate for each of the comparisons $\frac{1}{8}\left(b_{d 1}+\right.$ $\left.b_{d 2}+b_{d 3}+b_{d 4}-b_{d 5}-b_{d 6}-b_{d 7}-b_{d 8}\right)$ where the subscripts 1 to 8 relate to the $b_{d}$ from different substitution lines according to which comparison has been made. If we square this expression, we

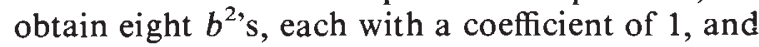
28 crossproducts among the $b$ 's, of which 12 have a coefficient of 2 and 16 have a coefficient of -2 , leaving a balance of 4 with a coefficient of -2 , and finally $1 / 64$ as a coefficient of the whole bracket. Thus the variance of the component of $c_{\mathrm{TL}}$ will be $(8 V-8 W) V_{E} / 64$ where $V$ relates to the relevant entry of the leading diagonal of the matrix (all of which are alike) and $W$ relates to the off-diagonal entries in the matrix, (all of which again are alike). Thus from the matrix in table $2(\mathrm{~b})$, the variance of each $c_{\mathrm{TL}}$ component will be $(29-9) V_{E} /(8 \times$ $252,000)$ which when $\times 10^{4}$ gives $V_{c}=1 \cdot 1519$ for $c_{\mathrm{TL} 1}$ where $V_{E}=11.611565$, and $V_{c}=2.4764$ for $c_{\mathrm{T} 2 \mathrm{~L}}$ where $V_{E}=2 \cdot 149790$, as we have seen. We may note that unlike $c_{\mathrm{LL}}$ and $c_{\mathrm{LT}}$ the variance of the means of the $c_{\mathrm{TL}}$ values is not the same as that of the orthogonal comparisons, since $b_{m}$ 's no longer cancel out.

Returning to the amounts of information, since the variances are expressed $\times 10^{4}$, the error variance of the orthogonal comparison of $c_{\mathrm{LL}}$ is 2.4479 , $I_{\mathrm{LL}}=0.40852$ i.e., the true value $\times 10^{-4}$. Similarly, $I_{\mathrm{LT} 1}=I_{\mathrm{LT} 2}=0.43405$ with $I_{\mathrm{T} 1 \mathrm{~L}}=0.86810$ and $I_{\mathrm{T} 2 \mathrm{~L}}=0 \cdot 40381$. These are the weights to be used in obtaining weighted least square estimates of $a$ and $r$ from $c_{\mathrm{LL}}, c_{\mathrm{LT}}$ and $c_{\mathrm{TL}}$. Their use will make but little difference where $T_{2}$ has been used as a tester, since the three amounts of information approximate quite closely to one another. This is not, however, the case where $T_{1}$ was the tester since $I_{\mathrm{T} 1 \mathrm{~L}}$ is over twice as large as $I_{\mathrm{LL}}$ and $I_{\mathrm{LT1}}$ : to ignore such a difference would result in inefficient estimates of $a$ and $r$, and hence to the possibility of misleading tests of significance.

The weighted least squares equations of estimation are

$$
\left(I_{\mathrm{LL}}+I_{\mathrm{TL}}\right) a+I_{\mathrm{LL}} r=I_{\mathrm{LL}} c_{\mathrm{LL}}+I_{\mathrm{TL}} c_{\mathrm{TL}}
$$


Table 5 The weighted least squares estimates of $a$ and $r$ for (above) the seven orthogonal comparisons among the substitution lines, and (below) the half-difference between the parental lines. The relevant standard error of the estimate ( $s$ ) is given at the foot of each column.

Significant values of $a$ and $r$ are indicated by ${ }^{*}$ where $0.05>P>0.01$ and ${ }^{*} *$ where $0.01>P$. Square brackets indicate the failure of the assumption that $a$ and $r$ are additive with $P<0.05$, and the round brackets indicate near failure with $P \approx 0 \cdot 10$

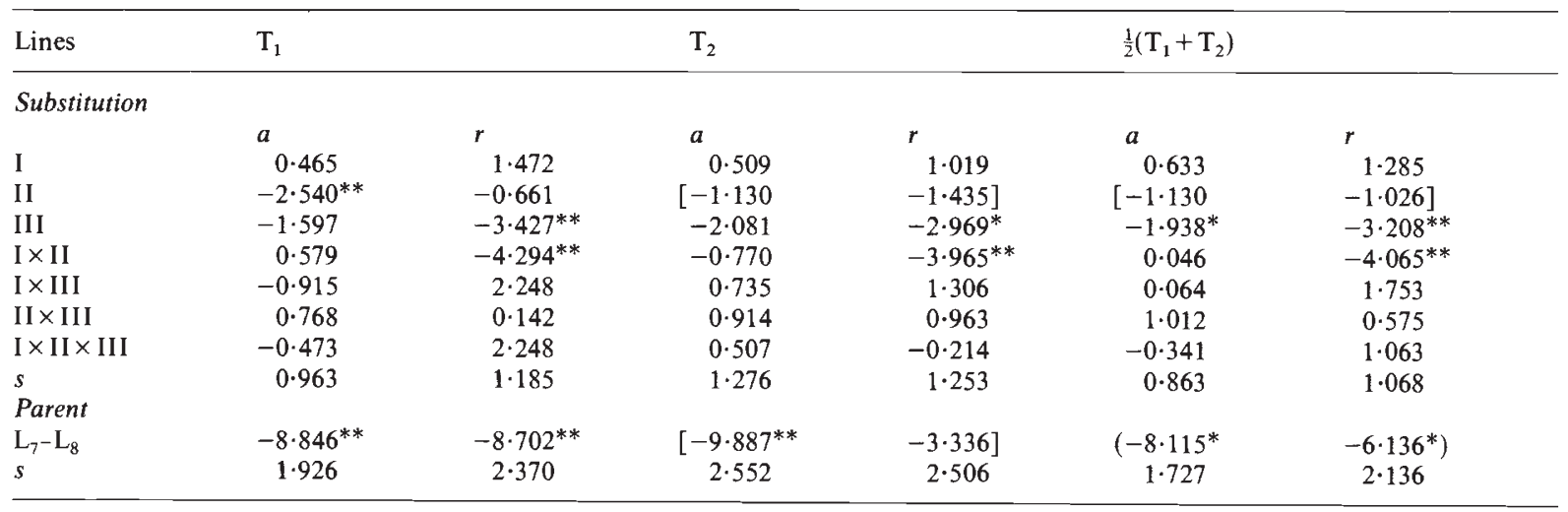

and

$$
I_{\mathrm{LL}} a+\left(I_{\mathrm{LL}}+I_{\mathrm{LT}}\right) r=I_{\mathrm{LL}} c_{\mathrm{LL}}+I_{\mathrm{LT}} c_{\mathrm{LT}}
$$

giving the information matrix

$$
\begin{aligned}
& {\left[\begin{array}{cc}
I_{\mathrm{LL}}+I_{\mathrm{LT}} & -I_{\mathrm{LL}} \\
-I_{\mathrm{LL}} & I_{\mathrm{LL}}+I_{\mathrm{TL}}
\end{array}\right]} \\
& \text { all } \div \mathrm{S}(I I)=I_{\mathrm{LL}} I_{\mathrm{LT}}+I_{\mathrm{LL}} I_{\mathrm{TL}}+I_{\mathrm{LT}} I_{\mathrm{TL}}
\end{aligned}
$$

and

$$
\begin{gathered}
V_{a}=\left(I_{\mathrm{LL}}+I_{\mathrm{LT}}\right) / S(I I), \quad V_{r}=\left(I_{\mathrm{LL}}+I_{\mathrm{TL}}\right) / \mathrm{S}(I I), \\
W_{a r}=-I_{\mathrm{LL}} / \mathrm{S}(I I) .
\end{gathered}
$$

The estimates of $a$ and $r$ obtained in this way for the seven orthogonal comparisons, using $T_{1}$ and $T_{2}$ in turn as the tester, are set out in the upper part of the table 5, and their standard errors are at the foot of each column. Significant values of $a$ and $r$ are marked by asterisks in the table. The results from the two tester lines are combined in the columns headed $\frac{1}{2}\left(T_{1}+T_{2}\right)$. These were obtained by starting the analysis with the means of $c_{\mathrm{LL}}, c_{\mathrm{LT}}$ and $c_{\mathrm{TL}}$ from the two tester lines, and deriving the estimates of $a$ and $r$ given in the $\frac{1}{2}\left(T_{1}+T_{2}\right)$ column by the same weighted least squares method of estimation. The value of $I_{\mathrm{LL}}$ for this combined estimation is obviously the same as that for the $T_{1}$ and $T_{2}$ analyses. The values of $c_{T L 1}$ and $c_{\mathrm{TL} 2}$ are derived from different sets of experimental data, and hence independent. $I_{\mathrm{TL}\left({ }_{2}^{1}\left[\mathrm{~T}_{1}+\mathrm{T}_{2}\right]\right)}$ is thus obtained as $\frac{1}{4}\left(V_{\mathrm{T} 1 \mathrm{~L}}+V_{\mathrm{T} 2 \mathrm{~L}}\right)$. The derivation of the joint $I_{\mathrm{LT}}$, is, however, more complex because the $c_{\mathrm{LT} 1}$ and $c_{\mathrm{LT} 2}$ for the same substitution line came from the same set of data. Hence $\frac{1}{2}\left(c_{\mathrm{LT} 1}+\right.$ $\left.c_{\mathrm{LT} 2}\right)=\frac{1}{2}\left(b_{d 1}+b_{d 2}-2 b_{m}\right)$ and the variance of the joint $c_{\mathrm{LT}}$ for each substitution line becomes $\frac{1}{4}(6 \mathrm{~V}-$ $6 W) V_{p a}$, where $V$ and $W$ again denote respectively the entries on and off the leading diagonal of the matrix in table $2(\mathrm{a})$. The variance of $\frac{1}{2}\left(c_{\mathrm{LT} 1}+c_{\mathrm{LT} 2}\right)$ is thus $3(17-9) V_{E} /(2 \times 100,800)$ for all the individual substitution lines and is one eighth of this for each of the orthogonal comparisons. Since the relevant $V_{E}=11.611565$ this becomes 1.727911 and $I_{\mathrm{LT}}=0.57873$ when multiplied and divided by $10^{4}$ respectively.

The analysis of the data obtained using $T_{1}$ as a tester confirms the findings from the analysis of the monocultures alone in that the only significant components of competition are those from the main effects of chromosomes I and III and the I $\times$ II interaction, but add the information that while the main effect of II is on $a$, III and I $\times$ II show effects on $r . T_{2}$ agrees with $T_{1}$ in showing significant effects on III and I $\times$ II on the value of $r$, and this is confirmed by the analysis of $\frac{1}{2}\left(T_{1}+T_{2}\right)$ with the addition of a smaller, but now significant effect of III on $a$. The effect of II on $a$ revealed by tester $T_{1}$ is not, however, confirmed when $T_{2}$ is used, and the $\frac{1}{2}\left(T_{1}+T_{2}\right)$ analysis agrees substantially with the $T_{2}$ analysis. The reason for this difference becomes clear when we use the data for a further test.

In estimating the values of $a$ and $r$, the assumption was made that they are additive. In each case three basic competition values, $c_{\mathrm{LL}}, c_{\mathrm{LT}}$ and $c_{\mathrm{TL}}$, were available for estimating the two parameters, 
and one of the three initial degrees of freedom remains available for testing the validity of this assumption of additivity. We proceed by finding $X=c_{\mathrm{LL}}-c_{\mathrm{LT}}-c_{\mathrm{TL}}$ which is expected to be zero apart from error variation if $a$ and $r$ can be treated as additive. Now $V_{x}=V_{c_{\mathrm{LL}}}+V_{c_{\mathrm{LT}}}+V_{c_{\mathrm{TL}}}$, and $X^{2} / V_{x}$ will be distributed as a $\chi_{1}^{2}$, testing the validity of the assumption of additivity on which the analysis was based. All seven of the orthogonal comparisons were tested in this way when $T_{1}, T_{2}$ and $\frac{1}{2}\left(T_{1}+T_{2}\right)$ were used as testers. In 19 out of the 21 cases this test gave no reason to doubt the validity of the assumption, but in the other two cases, the $\chi^{2}$ s were significant and the assumption of additivity must consequently be regarded as unsatisfactory. Both these cases relate to the components of competition mediated by the main effect of chromosome II, one of them being where $T_{2}$ was used as a tester, and the other, not surprisingly, were $\frac{1}{2}\left(T_{1}+T_{2}\right)$ was used. With $T_{1}$ as tester, this component of competition gave a perfectly good fit to the additivity model. Thus there must be interaction between aggression and response when $T_{2}$ is used as the tester, but not present when $T_{1}$ is so used.

The two parental lines, $\mathrm{L}_{7}$ and $\mathrm{L}_{8}$ were included in all the tests of the substitution lines, and the same kind of analysis of $c_{\mathrm{LL}}, c_{\mathrm{LT}}$ and $c_{\mathrm{TL}}$ used for the substitution lines can thus be carried out on the difference between these two lines. The only change is that when estimating the differences in $a$ and $r$ between $\mathrm{L}_{7}$ and $\mathrm{L}_{8}$, the variances will be four times as high, and the $I$ correspondingly have only one quarter the value. The results of the analysis of $L_{7}$ and $L_{8}$ are set out in the lower part of table 5 together with the standard errors of $a$ and $r$. The test of goodness of fit was also carried out in the same way as with the orthogonal comparisons, and we note that once again there is no good evidence of a departure from additivity of $a$ and $r$ when $\mathrm{T}_{1}$ is used as the tester, but the assumption of additivity fails with $\mathrm{T}_{2}$ just as it did for the main effect of chromosome II in the substitution lines. Evidently the interaction traced to the main effect of II by the analysis of the substitution lines is of not inconsiderable importance in the determination of the competitive relations between the genotypes of the parental lines $\mathrm{L}_{7}$ and $\mathrm{L}_{8}$.

Finally we may note that even when $T_{1}$ is used as the tester the sum of the effects on $a$ revealed by the different significant comparisons among the substitution lines is less than that revealed by the comparison between $\mathrm{L}_{7}$ and $\mathrm{L}_{8}$. Even with $r$ a similar though smaller shortfall is present. Here again we see the effects of genetic erosion in the construction of substitution lines.

\section{DISCUSSION AND CONCLUSIONS}

All three of the major chromosomes contribute to the mediation of competitive ability, and they do so in different ways. The only detectable difference between chromosome I of the two parental lines was in the interactive effect on the magnitude of response that it displayed with chromosome II: no effect on aggression was to be seen. Chromosome II had no significant effect on response beyond that stemming from an interaction with I, but had a clear effect on aggression. This however turned out to be displayed in monocultures and with $T_{1}$, but not when $\mathrm{T}_{2}$ was the responder. Chromosome III affected both response and, to a lesser extent, aggression, both effects being independent of the tester line used in their detection, and these effects have every appearance of being independent of each other. There is a hint from table 5 that there may be an interaction of III with I in the strength of its effect on response, but the evidence is by no means strong and it implies an interaction of the opposite sign to the clear $\mathrm{I} \times \mathrm{II}$ interaction. Nor does the effect of III on aggression show any sign of interaction with the other chromosomes.

Clearly the genetic determination of competitive ability is complex, and it is so not just in that genes in all the chrmosomes are involved but also in the way that they interact in exerting their effects. Chromosome III carries genes affecting both aggression and response, but there is no good evidence that the relevant genes interact either with genes in other chromosomes, or with one another. The gene differences on chromosome I on the other hand, show no main effect on either aggression or response, but they show a strong interaction with chromosome II on response (table 5). Furthermore, the difference in response produced by this I $\times$ II interaction appears to be the same whether the aggressor to which the substitution lines are responding is $T_{1}, T_{2}$ or the individual substitution lines themselves, for the values in the $c_{\mathrm{LL}}, c_{\mathrm{LT} 1}$ and $c_{\mathrm{LT} 2}$ columns in table 4 are alike within the limits of sampling variation. It is of particular interest that all are negative in sign. This shows that the parental combinations give lower response values than the recombinants. A lower $r$ value implies a smaller response to the competitive pressures and we are thus led to conclude that, at least in this case, flies with the parental combination of I and II are less affected by competitive pressure than are recombinants, that is that their genotypes are better balanced in this respect.

The $I \times I I$ interaction produces the same differences in response no matter what the genotype may be of the associate against which 
this response is tested. This interaction is thus the property of the genetical constitution and developmental processes of the individual itself: it is not affected by the genotypes of the individual with which it is associated. The component of the parental differences, reflecting the main effect of chromosome II is different. This II component can affect the value of $a$ as opposed to $r$, but shows a significant difference when the associate is $T_{1}$, and not when it is $T_{2}$ (table 5). Furthermore, when tested against $T_{2}$ the assumption of additivity fails. This failure is not, however, general: all the other six orthogonal components still accord with the assumption of an additive relation between $a$ and $r$, including the main effect of chromosome III which, as we have seen, covers the differences in both these parameters. Thus the behaviour of the substitution lines in duoculture with $T_{2}$ reveals an interaction not between genes in the genotype of single individuals, but between the relevant genes on chromosome II of the substitution lines and the genes in the genotype of the $T_{2}$ individuals. It implies an interaction between different genomes, not just an interaction between the products of genes within the same individual. Such intergenomic interaction affecting competitive ability could come about in a number of ways. It could arise as a result of individuals of different genic constitution using parts of the common environment in different ways as where larvae of the same laboratory population of Drosophila willistoni choose different places to pupate according to their genotypes (de Souza et al., 1968), so leading to different competitive pressures within and between the genotypes. Or it might spring from the inability of one genotype to use an essential feature of the environment in the absence of the other. This is well illustrated by the findings of Arthur (1986), albeit with a mixed population of Drosophila melanogaster and $D$. hydei. When the food medium is presented as a thin cake, $D$. hydei alone cannot prevent it from drying out and becoming unusable. But D. melanogaster can prevent it doing so with the result that $D$. hydei can also utilise the food in mixed culture with $D$. melanogaster. At the same time $D$. hydei is a strong competitor to $D$. melanogaster and so tends to reduce the numbers of the latter over the generations, the outcome being that the joint population settles down to being a stable mixture. Given an appropriate genic control of the differences in behaviour, there is no reason why such a situation should not arise within populations of a single species.

Inter-genomic interactions affecting competitive relationships can also arise in more directly biochemical ways, as noted by Mather and Caligari (1983). Drosophila melanogaster individuals cannot survive alone on a starch medium if they are homozygous for the allele $A m y^{\text {null }}$; but they do so if flies carrying the $A m y^{1}$ allele (which enables them to make amylase) are also present, because they obtain amylase or its products from the secretions of the $A m y^{1}$ flies via the medium, or other feature of the common environment ( $\mathrm{Haj}$ Ahmed and Hickey, 1982). The presence of flies carrying other Amy alleles of varying activity could account for intermediate levels of facilitation reported by De Jong and Scharloo (1975). At the same time competitive, as opposed to facilitative, interactions between individuals can be produced by secretion or excretion into the medium by individuals of one genotype of substances deleterious to other genotypes (Weisbrot, 1966). Indeed, density dependent polymorphism for alleles at the esterase locus can be maintained in this way (Huang et al., 1971; Kojima and Huang, 1972).

There is a further feature revealed by the present analysis which may possibly also be related to the behaviour of chromosome II. As noted earlier, in the surveys of the sixteen inbred lines and the monocultures of the present experiment, parental line 7 gave a $c_{m}=-b_{m}$ value which did not differ significantly from 0 , i.e., it showed no sign of intra-genotypic competition. Yet when the eight substitution lines were used as indicators with $T_{1}$ (which is $y^{2} 7$ ) as their associate, the mean value they gave for $c_{\mathrm{LT1}}$ was $-3 \cdot 3918$, which is significantly below $0\left(\chi_{1}^{2}=6 \cdot 26, P \approx 0 \cdot 01\right)$. The $c_{\mathrm{LT1}}$ for $L_{7}$ itself though small is not negative, but it does not depart significantly from the mean of $c_{\mathrm{LT} 1}$ of the substitution lines and if included with the values from the substitution lines, the overall mean is $-3 \cdot 2417$ which is still below 0 , though at a slightly reduced level of significance $\left(\chi_{1}^{2}=5 \cdot 131\right.$, $0.02<P<0.03)$. Such a negative value suggests that $T_{1}$ acts as an overall facilitator to $L_{7}$ and the substitution lines (see Mather and Caligari, 1981, 1983). Facilitation must imply an inter-genomic interaction, but further investigation would be required before this facilitation could be related to chromosome II, or even be regarded as established beyond reasonable doubt.

Earlier investigations showed that variation for competitive ability is present in the Texas cage population. The present analysis of the difference in competitive ability between two of the Texas inbred lines shows that all three major chromosomes are involved in the mediation of competitive interactions, and furthermore, that, at least in this 
case, they are involved in different ways. Chromosome III affects the levels of both aggression and response, but gives no evidence of their being interaction between the relevant genes. Chromosome I on the other hand, affects only response and that solely through an interaction with chromosome II, thus emphasising the prospective complexity of the determination of the level of response. Since this chromosome reveals no difference in effect on aggression, it also indicates that response and aggression are capable of independent adjustments by selection. This in turn implies that the genetical attributes of these two elements of competition may well turn out to be different. Indeed to the extent that it is revealed by the present assays, the architecture of response would appear to be more complex than that of aggression.

Nor need this surprise us if, as might be expected on the simplest view, the response to competitive pressure is a property of the individual. Whereas the competitive pressure exerted on it is potentially a property of the whole population and the many genotypes that it carries (Caligari and Mather, 1984). The behaviour of chromosome II warns us, however, that this view can be too simplistic, for the difference in effect between $L_{7}$ and $\mathrm{L}_{8}$ in respect of this chromosome was conditional on which tester line it was associated with, and was accompanied, as we have seen, by a breakdown of the simple assumption of additivity between $a$ and $r$, with in this case the difference in aggression vanishing. Thus the inter-genomic interaction here displays itself most obviously by a difference in the level of aggression; but a difference in the competitive pressures exerted by individuals of genotypes will be detected only if the indicator genotype has the ability of responding to such pressure under the circumstances of the experiment. Similarly, differences in response will be detected only if the associate has the capacity to exert competitive pressure under these circumstances-a relationship reminiscent of that between antigen and antibody.

The genetic determination and mechanism of the relationship between competitive pressure and response clearly can be complex. In the present case, the complexity may have been due, at least in part, to the parental lines, $\mathrm{L}_{7}$ and $\mathrm{L}_{8}$, having been chosen from among the available inbreds as being at opposite extremes in respect of their competitive abilities. Further elucidation may be obtainable by investigation of the differences between lines less extreme in competitive behaviour.

\section{REFERENCES}

ARTHUR, W. 1986. On the complexity of a simple environment: competition, resource partitioning and facilitation in a two-species Drosophila system. Phil. Trans. R. Soc. Lond. $B, 313,411-508$.

BARNES, B. W., AND KEARSEY, M. J. 1970. Variation for metrical characters in Drosophila populations. I. Genetic analysis. Heredity, 25, 1-10.

BIRLEY, A. J. 1984. Restriction endonuclease map variation and gene activity in the Adh region in a opulation of Drosophila melanogaster. Heredity, 52, 103-112.

BIRLEY, A. J., COUCH, P. A. AND MARSON, A. 1981. Genetical variation for enzyme activity in a population of Drosophila melanogaster. VI. Molecular variation in the control of alcohol dehydrogenase (ADH) activity. Heredity, 47, 185196.

CAlIGARI, P. D. S. 1980. Competitive interactions in Drosophila melanogaster. I. Monocultures. Heredity, 45, 219-231.

CALIGARI, P. D. S. AND MATHER, K. 1975. Genotype-environment interaction. III. Interactions in Drosophila melanogaster. Proc. R. Soc. Lond. B, 191, 387-411.

CALIGARI, P. D. S. AND MATHER, K. 1980. Dominance, allele frequency and selection in a population of Drosophila melanogaster. Proc. Roy. Soc. Lond. B., 208, 163-187.

CALIGARI, P. D. S. AND MATHER, K. 1984. Competitive interactions in Drosophila melanogaster. III. Triocultures. Heredity, 52, 255-264.

CALIGARI, P. D. S. AND POWELL, W. 1986. The effects of competition on variances and on seed germination in spring barley (Hordeum vulgare). Heredity, 57, 331-334.

DE JONG, G. AND SCHARLOO, w. 1975. Environmental determination of selective significance or neutrality of amylase variants in Drosophila melanogaster. Genetics, 84, 77-94.

EGGLESTON, P. 1985. Variation for aggression and response in the competitive interactions of Drosophila melanogaster. Heredity, 54, 43-51.

HAJ-AHMAD, Y. AND HICKEY, D. A. 1982. A molecular explanation of frequency-dependent selection in Drosophila. Nature Lond., 299, 350-352.

HILl, J., MATHER, K. AND CAligari, P. D. S. $1987 a$. Analysis of competitive ability among genotypes of perennial ryegrass: effect upon survival. Euphytica, 36, 99-107.

HILl, J., MATHER, K. AND CAligari, P. D. S. 1987 b. Analysis of competitive ability among genotypes of perennial ryegrass: effect upon dry matter production. Euphytica, 36, 109-115.

HUANG, S. L. SINGH, M. AND KOJIMA, K. 1971. A study of frequency-dependent selection observed in the esterase- 6 locus of Drosophila melanogaster using a conditioned media method. Genetics, 68, 97-104.

KEARSEY, M. J. AND BARNES, B. W. 1970. Variation for metrical characters in Drosophila populations. II. Natural selection Heredity, 25, 11-21.

KOJIMA, K. AND HUANG, S. L. 1972. Effects of population density on the frequency dependent selection in the esterase-6 locus of Drosophila melanogaster. Evolution, 26, 313-321.

MATHER, K. AND CALIGARI, P. D. S. 1981. Competitive interactions in Drosophila melanogaster. II. Measurement of competition. Heredity, 46, 239-254.

MATHER, K. AND CALIGARI, P. D. S. 1983. Pressure and response in competitive interactions. Heredity, 51, 435-454.

MATHER, K. AND HANKS, M. J. 1978. Genetics of coxal chaetae in Drosophila melanogaster. I. Variation in gene action. Heredity, 40, 71-96. 
MATHER, K., HILl, J. AND CALigari, P. D. S. 1982. Analysis of competitive ability among genotypes of perennial ryegrass. Heredity, 48, 421-434.

MINAWA, A. AND BIRLEY, A. J. 1978. The genetical response to natural selection by varied environment. I. Short-term observations. Heredity, 40, 39-50.

POWELL, W., CALIGARI, P. D. S., GOUDAPPEL, P. AND THOMAS, W. T. B. 1985. Competitive effects in monocultures and mixtures of spring barley. Theor. Appl. Genet., 71, 443-450.

SOUZA, H. L. DE, DA CUNHA, A. B. AND DOS SANTOS, E. P. 1968. Adaptive polymorphism of behaviour developed in laboratory populations of Drosophila willistoni. Amer. Nat., $102,583-586$.

WEISBROT, D. R. 1966. Genotypic interactions among competing strains and species of Drosophila. Genetics, 53, 427-435. 